\title{
RATIONALITY PROBLEM OF THREE-DIMENSIONAL PURELY MONOMIAL GROUP ACTIONS: THE LAST CASE
}

\author{
AKINARI HOSHI AND YŪICHI RIKUNA
}

\begin{abstract}
A $k$-automorphism $\sigma$ of the rational function field $k\left(x_{1}, \ldots, x_{n}\right)$ is called purely monomial if $\sigma$ sends every variable $x_{i}$ to a monic Laurent monomial in the variables $x_{1}, \ldots, x_{n}$. Let $G$ be a finite subgroup of purely monomial $k$-automorphisms of $k\left(x_{1}, \ldots, x_{n}\right)$. The rationality problem of the $G$-action is the problem of whether the $G$-fixed field $k\left(x_{1}, \ldots, x_{n}\right)^{G}$ is $k$-rational, i.e., purely transcendental over $k$, or not. In 1994, M. Hajja and M. Kang gave a positive answer for the rationality problem of the three-dimensional purely monomial group actions except one case. We show that the remaining case is also affirmative.
\end{abstract}

\section{INTRODUCTION}

Let $K$ be a field and $L$ a finite Galois extension of $k$. Let $\Pi$ be the Galois group of $L / K$ and $\mathcal{L}$ a $\Pi$-module with a $\mathbb{Z}$-free basis $\left\{l_{1}, \ldots, l_{n}\right\}$. Then an integral representation $\rho: \Pi \longrightarrow \mathbf{G L}_{n}(\mathbb{Z})$ is defined by $\sigma \longmapsto\left(a_{i j}\right)$ with

$$
l_{j}{ }^{\sigma}=\sum_{i=1}^{n} a_{i j} l_{i} \quad(1 \leq j \leq n) .
$$

We now assume that $\Pi$ acts on $L\left(x_{1}, \ldots, x_{n}\right)$, the rational function field over $L$ with $n$ variables $x_{1}, \ldots, x_{n}$, from the right by the following manner:

(1) $\Pi$ acts on $L$ as the Galois group,

(2) $x_{j}{ }^{\sigma}=\prod_{i=1}^{n} x_{i}{ }^{a_{i j}}$ with $\rho(\sigma)=\left(a_{i j}\right)$ for $1 \leq j \leq n$.

We know that there is a duality between the category of all $\Pi$-modules and the category of all algebraic $L / K$-tori, algebraic tori over $K$ which split over $L$. Then the fixed subfield $L\left(x_{1}, \ldots, x_{n}\right)^{\Pi}$ of $L\left(x_{1}, \ldots, x_{n}\right)$ can be identified with the function field of the algebraic $L / K$-torus $T$ corresponding to the $\Pi$-module $\mathcal{L}$ by the duality above. We say that the algebraic $L / K$-torus $T$ is rational when the $\Pi$-fixed field $L\left(x_{1}, \ldots, x_{n}\right)^{\Pi}$ is $K$-rational. One-dimensional algebraic tori are trivially rational. Voskresenskiı [17, 18] showed that all two-dimensional algebraic tori are rational. The birational classification of three-dimensional algebraic tori was given by Kunyavskiı $[8$. We note that there are many irrational algebraic tori of dimension $\geq 3$ (cf. [19]).

The rationality problem of a purely monomial group action is defined as a restricted version of "rationality questions" mentioned above. Let $k$ be a field and

Received by the editor December 8, 2006 and, in revised form, April 30, 2007.

2000 Mathematics Subject Classification. Primary 14E08, 12F12; Secondary 13A50, 14E07, $20 \mathrm{C} 10$. 
$k\left(x_{1}, \ldots, x_{n}\right)$ the function field over $k$ with $n$ variables $x_{1}, \ldots, x_{n}$. Let $G$ be a finite subgroup of $\mathbf{G L}_{n}(\mathbb{Z})$ which acts from the right on $k\left(x_{1}, \ldots, x_{n}\right)$ as follows:

(1) $G$ acts trivially on $k$,

(2) $x_{j}{ }^{A}=\prod_{i=1}^{n} x_{i}{ }^{a_{i j}}$ with $A=\left(a_{i j}\right) \in G$ for $1 \leq j \leq n$.

We call the $G$-action purely monomial. The rationality problem of the purely monomial $G$-action is the problem of whether the fixed subfield $k\left(x_{1}, \ldots, x_{n}\right)^{G}$ of $k\left(x_{1}, \ldots, x_{n}\right)$ is $k$-rational or not.

The fixed field of a purely monomial group action generally cannot be identified with a function field of any algebraic torus. But the rationality problem of purely monomial group actions has a special meaning in constructive aspects of inverse Galois theory. Let $\Gamma$ be a finite group acting on the rational function field $k\left(x_{g} \mid g \in \Gamma\right)$ via the regular representation. The $k$-rationality problem of this $\Gamma$-action is called Noether's problem of $\Gamma$ over $k$. If this problem has a positive answer, we can construct a regular Galois $\Gamma$-extension over $k\left(x_{g} \mid g \in \Gamma\right)^{\Gamma}$. This is known as Noether's strategy for constructing a generic Galois $\Gamma$-extension over $k$. When $\Gamma$ is abelian, Lenstra 9] gave a necessary and sufficient condition that the Noether's problem of $\Gamma$ over $k$ has a positive answer. We, however, know very little for non-abelian cases. The rationality problem of purely monomial group actions is crucial in studying Noether's problem of non-abelian groups. The reader may consult [5, 6, 12, 13, 14, 15] about Noether's problem.

The rationality problem of one-dimensional purely monomial group actions is trivially affirmative. For two-dimensional cases, Hajja 2] gave the following result:

Theorem 1.1 (Hajja). Let $k$ be a field and $G$ be a finite subgroup of $\mathbf{G L}_{2}(\mathbb{Z})$. Then $k\left(x_{1}, x_{2}\right)^{G}$ is k-rational.

The three-dimensional cases are much more difficult than the two-dimensional ones. Tahara 16 proved that $\mathbf{G L}_{3}(\mathbb{Z})$ has 73 conjugacy classes of finite subgroups. Hajja-Kang 3, 4, obtained affirmative answers for 72 classes of them. Let $G_{0}$ be the finite subgroup of $\mathbf{G L}_{3}(\mathbb{Z})$ generated by

$$
\left(\begin{array}{ccc}
1 & 1 & 0 \\
-2 & -1 & -1 \\
0 & 0 & 1
\end{array}\right) \text { and }\left(\begin{array}{ccc}
-1 & -1 & -1 \\
0 & 0 & 1 \\
0 & 1 & 0
\end{array}\right)
$$

Theorem 1.2 (Hajja-Kang). Let $k$ be a field and $G$ be a finite subgroup of $\mathbf{G L}_{3}(\mathbb{Z})$. Then $k\left(x_{1}, x_{2}, x_{3}\right)^{G}$ is $k$-rational if $G$ is not conjugate to $G_{0}$ in $\mathbf{G L}_{3}(\mathbb{Z})$.

A three-dimensional algebraic torus corresponding to $G_{0}$ (or its congugate in $\mathbf{G L}_{3}(\mathbb{Z})$ ) is not rational. For this reason, it might have been considerd that the remaining case is negative. But it is also a fact that there are irrational threedimensional algebraic tori corresponding to purely monomial group actions whose fixed fields are $k$-rational. In this paper, we show that the remaining case is also affirmative.

Theorem 1.3 (Main result). For an arbitrary field $k$, the fixed field $k\left(x_{1}, x_{2}, x_{3}\right)^{G_{0}}$ is $k$-rational. Consequently, the rationality problem of the three-dimensional purely monomial group actions has a positive answer.

Finally, we note that this result can also be expressed from a viewpoint of multiplicative invariant theory. The lattice which is treated in the main result is isomorphic to the signed root lattice $\mathbb{Z}^{-} \otimes_{\mathbb{Z}} A_{3}$. The rationality problem for this lattice 
is introduced as an interesting open problem in [10, Problem 14]. Let $\mathfrak{S}_{n}$ be the symmetric group on $n$ letters $\{1, \ldots, n\}$. The group $\mathfrak{S}_{n}$ acts multiplicatively on $\mathbb{Z}$ via the sign homomorphism. We denote the non-trivial $\mathfrak{S}_{n}$-lattice with this action by $\mathbb{Z}^{-}$, and we regard $\mathbb{Z}$ as the trivial lattice. For $n \geq 2, \mathfrak{S}_{n}$ permutes a $\mathbb{Z}$-basis of the lattice $\mathbb{Z}\left[\mathfrak{S}_{n} / \mathfrak{S}_{n-1}\right]$. The kernel $A_{n-1}$ of the augmentation map of the permutation $\mathfrak{S}_{n}$-lattice $\mathbb{Z}\left[\mathfrak{S}_{n} / \mathfrak{S}_{n-1}\right]$ also has a $\mathfrak{S}_{n}$-lattice structure. Thus we obtain a signed root lattice $\mathbb{Z}^{-} \otimes_{\mathbb{Z}} A_{n-1}$. The $k$-rationality problem of $k\left(\mathbb{Z}^{-} \otimes_{\mathbb{Z}} A_{3}\right)^{\mathfrak{S}_{4}}$ is equivalent to Hajja-Kang's "the exceptional case" treated as $W_{10}(198)$ in [4].

Corollary 1.4. For an arbitrary field $k$, the $\mathfrak{S}_{4}$-invariant field $k\left(\mathbb{Z}^{-} \otimes_{\mathbb{Z}} A_{3}\right)^{\mathfrak{S}_{4}}$ is $k$-rational.

\section{StRATEGy}

Our purpose is to show $k\left(x_{1}, x_{2}, x_{3}\right)^{G_{0}}$, where $G_{0}=\left\langle A_{0}, B_{0}\right\rangle$ with

$$
A_{0}:=\left(\begin{array}{ccc}
1 & 1 & 0 \\
-2 & -1 & -1 \\
0 & 0 & 1
\end{array}\right), \quad B_{0}:=\left(\begin{array}{ccc}
-1 & -1 & -1 \\
0 & 0 & 1 \\
0 & 1 & 0
\end{array}\right),
$$

is rational over an arbitrary field $k$. From a relation $A_{0}{ }^{4}=B_{0}{ }^{2}=\left(A_{0} B_{0}\right)^{3}=I_{3}$, where $I_{3}$ is the identity matrix, $G_{0}$ is isomorphic to the symmetric group $\mathfrak{S}_{4}$. Here we put

$$
A_{1}:=B_{0} A_{0}^{2}=\left(\begin{array}{ccc}
1 & 1 & 0 \\
0 & 0 & 1 \\
0 & -1 & 0
\end{array}\right), \quad B_{1}:=A_{0} B_{0} A_{0}^{2}=\left(\begin{array}{ccc}
1 & 1 & 1 \\
-2 & -1 & -1 \\
0 & -1 & 0
\end{array}\right) ;
$$

then $G_{0}$ is also generated by $A_{1}$ and $B_{1}$. To simplify our calculations, we take $G:=\left\langle P^{-1} A_{1} P, P^{-1} B_{1} P\right\rangle$ where

$$
P:=\left(\begin{array}{ccc}
-1 & -1 & -1 \\
0 & 1 & 0 \\
1 & 0 & 0
\end{array}\right) \in \mathbf{G L}_{3}(\mathbb{Z})
$$

Because $G_{0}$ and $G$ are conjugate in $\mathbf{G L}_{3}(\mathbb{Z})$, it is enough to show the $k$-rationality of the $G$-action to prove Theorem 1.3 .

Denote $P^{-1} A_{1} P$ and $P^{-1} B_{1} P$ by $A$ and $B$ respectively:

$$
A:=\left(\begin{array}{ccc}
0 & -1 & 0 \\
1 & 0 & 0 \\
0 & 1 & 1
\end{array}\right), \quad B:=\left(\begin{array}{ccc}
0 & -1 & 0 \\
1 & 1 & 2 \\
-1 & 0 & -1
\end{array}\right) .
$$

They satisfy $A^{4}=B^{3}=\left(B^{-1} A^{2} B\right)^{2}=(A B)^{2}=I_{3}$. Hence $G$ has the following normal series:

$$
1 \quad \triangleleft \quad\left\langle A^{2}\right\rangle \quad \triangleleft \quad\left\langle A^{2}, B^{-1} A^{2} B\right\rangle \quad \triangleleft \quad\langle A, B\rangle=G .
$$

We first choose appropriate $\left\langle A^{2}\right\rangle$-invariant functions $s_{1}, s_{2}, s_{3}$ which generate the $\left\langle A^{2}\right\rangle$-fixed field $k\left(x_{1}, x_{2}, x_{3}\right)^{\left\langle A^{2}\right\rangle}$ over $k$. To do this, we use the following lemma concerning two-dimensional weighted diagonal involutions which was obtained by Hajja-Kang [4]: 
Lemma 2.1 (Hajja-Kang). Let $k$ be a field and $\sigma \in \mathbf{A u t}_{k} k\left(x_{1}, x_{2}\right)$ be an involution defined by $\left(x_{1}, x_{2}\right) \longmapsto\left(m_{1} / x_{1}, m_{2} / x_{2}\right)$ with $m_{1}, m_{2} \in k^{\times}$. Then the fixed field $k\left(x_{1}, x_{2}\right)^{\langle\sigma\rangle}$ is

$$
k\left(\frac{x_{1}^{2} x_{2}^{2}-m_{1} m_{2}}{x_{1}\left(x_{2}^{2}-m_{2}\right)}, \frac{x_{2}\left(x_{1}^{2}-m_{1}\right)}{x_{1}\left(x_{2}^{2}-m_{2}\right)}\right) .
$$

Since $\left\langle A^{2}\right\rangle$ has index two in $\left\langle A^{2}, B^{-1} A^{2} B\right\rangle$, we can find $\left\langle A^{2}, B^{-1} A^{2} B\right\rangle$-invariant functions $t_{1}, t_{2}, t_{3}$ which generate the $\left\langle A^{2}, B^{-1} A^{2} B\right\rangle$-fixed field. Finally, we show that the purely monomial $\langle A, B\rangle$-action on $k\left(t_{1}, t_{2}, t_{3}\right)$ is $k$-rational. We can find new generators $u_{1}, u_{2}, u_{3}$ of $k\left(t_{1}, t_{2}, t_{3}\right)$ to apply the following lemma given by Ahmad-Hajja-Kang [1, Theorem. 3.1].

Lemma 2.2 (Ahmad-Hajja-Kang). Let $L$ be an arbitrary field and $L(x)$ be the rational function field with one variable over $L$. Let $H$ be a group of automorphisms acting on $L(x)$. Suppose that, for any $\sigma \in H, \sigma(L) \subset L, x^{\sigma}=a_{\sigma} x+b_{\sigma}$ for some $a_{\sigma} \in L \backslash\{0\}$ and $b_{\sigma} \in L$. Then $L(x)=L^{H}$ or $L^{H}(f(x))$ for some polynomial $f(x) \in L[x]$ with positive degree. In particular, if $L^{H}$ is rational over some subfield $M$, so is $L(x)^{H}$ over $M$.

The final step is easier when the characteristic of $k$ is two.

\section{Proof of Theorem 1.3}

The action of $G=\langle A, B\rangle$ on $k\left(x_{1}, x_{2}, x_{3}\right)$ is described by

$$
\begin{cases}A: & \left(x_{1}, x_{2}, x_{3}\right) \longmapsto\left(x_{2}, x_{3} / x_{1}, x_{3}\right), \\ B: & \left(x_{1}, x_{2}, x_{3}\right) \longmapsto\left(x_{2} / x_{3}, x_{2} / x_{1}, x_{2}{ }^{2} / x_{3}\right) .\end{cases}
$$

3.1. The case when the characteristic of $k$ is not two. The action of $A^{2}$ on $k\left(x_{1}, x_{2}, x_{3}\right)$ is

$$
\left(x_{1}, x_{2}, x_{3}\right) \longmapsto\left(x_{3} / x_{1}, x_{3} / x_{2}, x_{3}\right) .
$$

From Lemma 2.1. the fixed field $k\left(x_{1}, x_{2}, x_{3}\right)^{\left\langle A^{2}\right\rangle}$ is $k\left(s_{1}{ }^{\prime}, s_{2}{ }^{\prime}, s_{3}{ }^{\prime}\right)$, where

$$
s_{1}{ }^{\prime}:=\frac{x_{1}{ }^{2} x_{2}{ }^{2}-x_{3}{ }^{2}}{x_{1}\left(x_{2}{ }^{2}-x_{3}\right)}, \quad s_{2}{ }^{\prime}:=\frac{x_{2}\left(x_{1}{ }^{2}-x_{3}\right)}{x_{1}\left(x_{2}{ }^{2}-x_{3}\right)}, \quad s_{3}{ }^{\prime}:=x_{3} .
$$

Then $B^{-1} A^{2} B$ acts on $k\left(s_{1}{ }^{\prime}, s_{2}{ }^{\prime}, s_{3}{ }^{\prime}\right)$ by

$$
\left(s_{1}{ }^{\prime}, s_{2}{ }^{\prime}, s_{3}{ }^{\prime}\right) \longmapsto\left(\frac{\left(1-s_{2}{ }^{\prime}\right)\left(1+s_{2}{ }^{\prime}\right)}{s_{1}{ }^{\prime}},-s_{2}{ }^{\prime}, \frac{1}{s_{3}{ }^{\prime}}\right) \text {. }
$$

To linearize this action, we take the following birational transformation over $k$ :

$$
s_{1}:=\frac{s_{1}{ }^{\prime}+\left(1+s_{2}{ }^{\prime}\right)}{s_{1}{ }^{\prime}-\left(1+s_{2}{ }^{\prime}\right)}, \quad s_{2}:=\frac{s_{1}{ }^{\prime}+s_{3}{ }^{\prime}\left(1+s_{2}{ }^{\prime}\right)}{s_{1}{ }^{\prime}-s_{3}{ }^{\prime}\left(1+s_{2}{ }^{\prime}\right)}, \quad s_{3}:=s_{2}{ }^{\prime} .
$$

Then we have $k\left(s_{1}{ }^{\prime}, s_{2}{ }^{\prime}, s_{3}{ }^{\prime}\right)=k\left(s_{1}, s_{2}, s_{3}\right)$ and

$$
B^{-1} A^{2} B: \quad\left(s_{1}, s_{2}, s_{3}\right) \longmapsto\left(-s_{1},-s_{2},-s_{3}\right) .
$$

We have $k\left(s_{1}, s_{2}, s_{3}\right)^{\left\langle B^{-1} A^{2} B\right\rangle}=k\left(t_{1}{ }^{\prime}, t_{2}{ }^{\prime}, t_{3}{ }^{\prime}\right)$ where

$$
t_{1}{ }^{\prime}:=s_{1} s_{3}, \quad t_{2}{ }^{\prime}:=s_{2} s_{3}, \quad t_{3}{ }^{\prime}:=s_{3}{ }^{2} \text {. }
$$


The action of $B$ on $k\left(t_{1}{ }^{\prime}, t_{2}{ }^{\prime}, t_{3}{ }^{\prime}\right)$ is described as

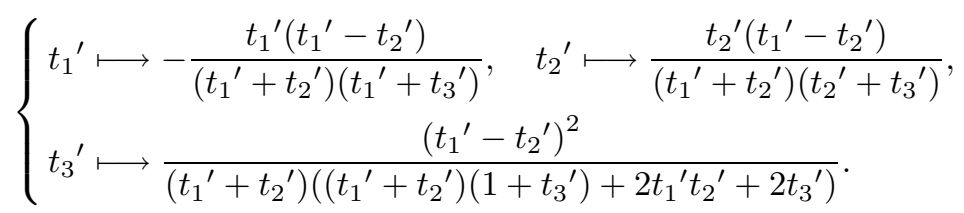

We observe that (3.8) has a symmetry with respect to $t_{1}{ }^{\prime}$ and $t_{2}{ }^{\prime}$. By using this property, we put

$$
t_{1}:=\frac{t_{1}{ }^{\prime}-t_{2}{ }^{\prime}}{t_{1}{ }^{\prime}+t_{2}{ }^{\prime}}, \quad t_{2}:=\frac{2 t_{1}{ }^{\prime} t_{2}{ }^{\prime}+\left(t_{1}{ }^{\prime}+t_{2}{ }^{\prime}\right) t_{3}{ }^{\prime}}{\left(t_{1}{ }^{\prime}-t_{2}{ }^{\prime}\right) t_{3}{ }^{\prime}}, \quad t_{3}:=\frac{t_{1}{ }^{\prime}+t_{2}{ }^{\prime}+2 t_{1}{ }^{\prime} t_{2}{ }^{\prime}}{t_{1}{ }^{\prime}-t_{2}{ }^{\prime}}
$$

to linearize the $\langle A, B\rangle$-action on $k\left(t_{1}{ }^{\prime}, t_{2}{ }^{\prime}, t_{3}{ }^{\prime}\right)$. This is a birational transformation, because we have

$$
t_{1}{ }^{\prime}=\frac{1-t_{1} t_{3}}{1-t_{1} t_{2}}, \quad t_{2}{ }^{\prime}=\frac{1-t_{1} t_{3}}{-1+t_{1}}, \quad t_{3}{ }^{\prime}=\frac{-1+t_{1} t_{3}}{1+t_{1}} .
$$

Hence $k\left(t_{1}, t_{2}, t_{3}\right)=k\left(t_{1}^{\prime}, t_{2}^{\prime}, t_{3}^{\prime}\right)$, and the $\langle A, B\rangle$-action is described as follows:

$$
\begin{cases}A: & \left(t_{1}, t_{2}, t_{3}\right) \longmapsto\left(-t_{1},-t_{3},-t_{2}\right), \\ B: & \left(t_{1}, t_{2}, t_{3}\right) \longmapsto\left(t_{2}, t_{3}, t_{1}\right) .\end{cases}
$$

We finally put

$$
u_{1}:=t_{2} / t_{1}, \quad u_{2}:=t_{3} / t_{1}, \quad u_{3}:=t_{1},
$$

and hence $k\left(t_{1}, t_{2}, t_{3}\right)=k\left(u_{1}, u_{2}, u_{3}\right)$,

$$
\begin{cases}A: & \left(u_{1}, u_{2}, u_{3}\right) \longmapsto\left(u_{2}, u_{1},-u_{3}\right), \\ B: & \left(u_{1}, u_{2}, u_{3}\right) \longmapsto\left(u_{2} / u_{1}, 1 / u_{1}, u_{1} u_{3}\right) .\end{cases}
$$

For $L:=k\left(u_{1}, u_{2}\right)$, we can easily check the following properties:

(1) $\sigma(L) \subset L$ for every $\sigma \in\langle A, B\rangle$.

(2) For any $\sigma \in\langle A, B\rangle, u_{3}{ }^{\sigma}$ has degree one in $L\left[u_{3}\right]$.

(3) $L\left(u_{3}\right)^{\langle A, B\rangle} \neq L^{\langle A, B\rangle}$.

Therefore we can apply Lemma 2.2 to $L\left(u_{3}\right)^{\langle A, B\rangle}$. This follows that the $G$-fixed field $k\left(x_{1}, x_{2}, x_{3}\right)^{G}$ is rational over $k$.

3.2. The case when the characteristic of $k$ is two. We recall (3.3); then $k\left(x_{1}, x_{2}, x_{3}\right)^{\left\langle A^{2}\right\rangle}$ is generated by $s_{1}{ }^{\prime}, s_{2}{ }^{\prime}, s_{3}{ }^{\prime}$ over $k$. Put

$$
s_{1}:=\frac{1+s_{2}{ }^{\prime}}{s_{1}{ }^{\prime}}, \quad s_{2}:=\frac{\left(1+s_{2}{ }^{\prime}\right) s_{3}{ }^{\prime}}{s_{1}{ }^{\prime}}, \quad s_{3}:=s_{2}{ }^{\prime}
$$

so that $k\left(s_{1}{ }^{\prime}, s_{2}{ }^{\prime}, s_{3}{ }^{\prime}\right)=k\left(s_{1}, s_{2}, s_{3}\right)$ and

$$
B^{-1} A^{2} B: \quad\left(s_{1}, s_{2}, s_{3}\right) \longmapsto\left(1 / s_{1}, 1 / s_{2}, s_{3}\right) .
$$

Applying Lemma 2.1 to $k\left(s_{1}, s_{2}, s_{3}\right)$, we have $k\left(s_{1}, s_{2}, s_{3}\right)^{\left\langle B^{-1} A^{2} B\right\rangle}=k\left(t_{1}{ }^{\prime}, t_{2}{ }^{\prime}, t_{3}{ }^{\prime}\right)$ where

$$
t_{1}{ }^{\prime}:=\frac{s_{1}{ }^{2} s_{2}{ }^{2}-1}{s_{1}\left(s_{2}{ }^{2}-1\right)}, \quad t_{2}{ }^{\prime}:=\frac{s_{2}\left(s_{1}{ }^{2}-1\right)}{s_{1}\left(s_{2}{ }^{2}-1\right)}, \quad t_{3}{ }^{\prime}:=s_{3} .
$$


Then we obtain

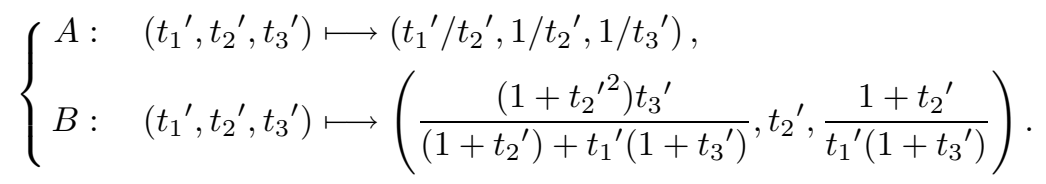

We here take

$$
t_{1}:=\frac{1+t_{2}{ }^{\prime}}{t_{1}{ }^{\prime}\left(1+t_{3}{ }^{\prime}\right)}, \quad t_{2}:=\frac{t_{1}{ }^{\prime}\left(1+t_{3}{ }^{\prime}\right)}{t_{3}{ }^{\prime}\left(1+t_{2}{ }^{\prime}\right)}, \quad t_{3}:=t_{2}{ }^{\prime}
$$

so that the $\langle A, B\rangle$-action is purely monomial. Then we can check $k\left(t_{1}{ }^{\prime}, t_{2}{ }^{\prime}, t_{3}{ }^{\prime}\right)=$ $k\left(t_{1}, t_{2}, t_{3}\right)$ and that $\langle A, B\rangle$ acts on $k\left(t_{1}, t_{2}, t_{3}\right)$ by

$$
\begin{cases}A: & \left(t_{1}, t_{2}, t_{3}\right) \longmapsto\left(1 / t_{2}, 1 / t_{1}, 1 / t_{3}\right), \\ B: & \left(t_{1}, t_{2}, t_{3}\right) \longmapsto\left(t_{2}, 1 / t_{1} t_{2}, t_{3}\right) .\end{cases}
$$

This is a purely monomial $\mathfrak{S}_{3}$-action. Theorem 1.2 shows that $k\left(t_{1}, t_{2}, t_{3}\right)^{\langle A, B\rangle}$ is $k$-rational. This completes the proof of Theorem 1.3 .

Remark 3.1. It is possible to compute explicit generators of $k\left(x_{1}, x_{2}, x_{3}\right)^{G}$ over $k$ with any characteristic by continuing the method above. To do this, one can use the explicit positive result about the Noether's problem of the cyclic group of order three in Kuniyoshi [7] and Masuda [1]. We omit displaying them because of their complicated expressions.

\section{ACKNOWLEDGEMENT}

The authors would like to thank the referee who provided them with some historical remarks and very kind advice on making their proof simpler. This study was supported by Grant-in-Aid for JSPS (the Japan Society for the Promotion of Science) Fellows.

\section{REFERENCES}

1. H. Ahmad, M. Hajja, and M. Kang, Rationality of some projective linear actions, J. Algebra 228 (2000), no. 2, 643-658. MR.1764585 (2001e:12003)

2. M. Hajja, Rationality of finite groups of monomial automorphisms of $k(x, y)$, J. Algebra 109 (1987), no. 1, 46-51. MR898335 (88j:12002)

3. M. Hajja and M. Kang, Finite group actions on rational function fields, J. Algebra 149 (1992), no. 1, 139-154. MR1165204 (93d:12009)

4. M. Hajja and M. Kang, Three-dimensional purely monomial group actions, J. Algebra 170 (1994), no. 3, 805-860. MR1305266 (95k:12008)

5. C. U. Jensen, A. Ledet, and N. Yui, Generic polynomials, Constructive aspects of the inverse Galois problem, Mathematical Sciences Research Institute Publications, vol. 45, Cambridge University Press, Cambridge, 2002. MR1969648 (2004d:12007)

6. I. Kersten, Noether's problem and normalization, Jahresber. Deutsch. Math.-Verein. 100 (1998), no. 1, 3-22. MR1617295 (99h:12003)

7. H. Kuniyoshi, On a problem of Chevalley, Nagoya Math. J. 8 (1955), 65-67. MR0069160 (16:993d)

8. B. Ė. Kunyavskiǔ, Three-dimensional algebraic tori, Translated in Selecta Math. Soviet. 9 (1990), no. 1, 1-21. MR.1032541 (91g:14050)

9. H. W. Lenstra, Rational functions invariant under a finite abelian group, Invent. Math. 25 (1974), 299-325. MR0347788 (50:289)

10. M. Lorenz, Multiplicative invariant theory, Encyclopaedia of Mathematical Sciences vol. 135, Invariant Theory and Algebraic Transformation Groups VI. Springer-Verlag, Berlin, 2005. MR:2131760 (2005m:13012) 
11. K. Masuda, On a problem of Chevalley, Nagoya Math. J. 8 (1955), 59-63. MR0069159 (16:993c)

12. E. Noether, Gleichungen mit vorgeschriebener Gruppe, Math. Ann. 78 (1918), 221-229. MR.1511893

13. J.-P. Serre, Topics in Galois theory, Research Notes in Mathematics, vol. 1, Jones and Bartlett Publishers, Boston, MA, 1992. MR1162313(94d:12006)

14. J.-P. Serre, Cohomological invariants, Witt invariants, and trace forms, Notes by Skip Garibaldi. Univ. Lecture Ser., 28, Cohomological invariants in Galois cohomology, 1-100, Amer. Math. Soc., Providence, RI, 2003. MR1999384

15. R. G. Swan, Noether's problem in Galois theory, Emmy Noether in Bryn Mawr (Bryn Mawr, Pa., 1982), 21-40, Springer, New York-Berlin, 1983. MR713790 (84k:12013)

16. K. Tahara, On the finite subgroups of $\mathrm{GL}(3, \mathbb{Z})$, Nagoya Math. J. 41 (1971), 169-209. MR 0272910 (42:7791)

17. V. E. Voskresenskiǔ, On two-dimensional algebraic tori, Izv. Akad. Nauk SSSR Ser. Mat. 29 (1965), 239-244. MR0172881 (30:3097)

18. V. E. Voskresenskiǔ, On two-dimensional algebraic tori II, Izv. Akad. Nauk SSSR Ser. Mat. 31 (1967), 711-716. MR0214597 (35:5446)

19. V. E. Voskresenskiū, Algebraic groups and their birational invariants, Translations of Mathematical Monographs, vol. 179. American Mathematical Society, Providence, RI, 1998. MR:1634406 (99g:20090)

Department of Mathematics, School of Education, Waseda University, 1-6-1 NishiWaseda Shinjuku-Ku, Tokyo 169-8050, JaPAn

E-mail address: hoshi@ruri.waseda.jp

Department of Applied Mathematics, School of Fundamental Science and Engineering, Waseda University, 3-4-1 Ohkubo Shinjuku-ku, Tokyo 169-8555, Japan

E-mail address: rikuna@moegi.waseda.jp 